\title{
Regulatory and Metabolic Interactions of Carbohydrates and Lipids in Diabetes and Obesity
}

\author{
Dr. Chrysanthus Chukwuma Sr \\ Executive Director, Centre for Future-Oriented Studies, Abakaliki, Ebonyi State, Nigeria
}

\begin{abstract}
This paper attempts to review available pertinent scientific and medical literature that the current global epidemic of obesity and diabetes has increased simultaneously or contemporaneously with adverse metabolic events. There is widespread established evidence that the type of carbohydrate consumed is important in the development or prevention of insulin resistance, obesity, and the metabolic syndrome. There is scarcity of published data on the combined effects of interactions between micronutrients, carbohydrates and lipids. However, there are extensive data supporting the benefits of the effects of both carbohydrates and lipids on various mechanisms of energy balance and diseases. Adequate measure is necessary to control carbohydrate-lipid interactions as they impact on obesity and diabetes.
\end{abstract}

Keywords: Management; Global; Etiopathogenesis; Control; Diet; Epidemic.

(9) (i) CC BY: Creative Commons Attribution License 4.0

\section{Introduction}

The exponential increase of chronic diseases, such as diabetes and obesity is predicted to escalate exponentially with co-existence of the two diseases and diverse organ disorders globally in the human population. Obesity is increasing at an astronomic proportion globally, and the encompassing consequence of obesity on several organ systems in the human body is currently given due cognizance [1]. The presenting epidemic of obesity and type 2 diabetes globally has brought to the fore on the metabolic perturbations critical to aetiological events. Diet is a potent functionality in modulating metabolic syndrome expression that is associated with the levels and varieties of carbohydrates and fats as well as their interactions as significant parameters. Insulin resistance is significant in the metabolic syndrome due to relative insulin failure to govern the numerous biological effects on the metabolism of carbohydrates and lipids. There is paucity of published data on the combined impacts on interaction between the micronutrients, dietary fats and carbohydrate varieties. There are extant expansive data undergirding the advantageous impact of both fat and carbohydrates moieties on diverse mechanisms of energy balance, metabolism and disorders. Certain mechanisms depict how the quality of dietary carbohydrate can affect weight gain by the absorption rate or the magnitude of carbohydrate fermentation.

Evidence abound indicating that the carbohydrate type is significant to developing or preventing insulin resistance, obesity, and the metabolic syndrome which need to be taken into cognizance to prevent, control and treat the presenting condition [2,3]. Diabetes constitute a metabolic disorder characterized by chronic hyperglycaemia and immune-mediated type 1 diabetes [3], insulin-resistant type 2 diabetes, gestational, genetic, environmental, infectious, behavioural or drug-induced impairments [4], and in syndemics or co-morbidity relationship with obesity $[5,6]$. In the evolutionary process, the adaptation of living organisms has been associated with utilization of diverse nutrient sources, such as carbohydrates, lipids and proteins. However, carbohydrates and lipids constitute the two principal macromolecular components of intracellular storage compounds for energy production. Significantly, the anabolism and catabolism of these macromolecules are inextricably linked in organs concerned with nutrient regulation, such as the brain, liver, adipose tissue, pancreas and muscle [7]. The rate of obesity has been exacerbated globally, thus imposing on already augmented social burden regarding quality of life.

\section{Etiopathogenesis}

It has been difficult to define a clear trajectory for carbohydrate and lipid metabolism including their extant but elusive interactions. It has been determined that plants and animals conserve lipids from carbohydrates, but the reverse is evidenced in plants and, not practically imminent in animals [8]. In conventional human metabolism, carbohydrate-lipid interactions are not clearly evident, but the perturbations extant in diabetic metabolism manifest these interactions. Carbohydrate or lipid is utilizable as the major source of body fuel, however, the metabolic trajectory varies in chemical processes and functionalities. It is evident in the low content of body carbohydrate, such as in severe debilitative diabetes and starvation that lipids make provision for body fuel, and it is also exhibited in the blood and internal organs, particularly the hepatic organ [4, 8]. On recognition that there is abysmal decrease of carbohydrate necessary for metabolism, depot lipid is received by the hepatic organ and catabolized to ketone bodies, and peripherally burned in the muscles exclusive of carbohydrate metabolism [7]. This presents as a normal process, but inimical solely in diabetes. Diabetic obesity is frequently observed at the inception and immediately following insulin treatment. Ostensibly, hyperglycaemia induces this obesity that has been determined in unique presentations of lipaemia, diabetes and lipodystrophy. Lipaemia is obvious in two opposite metabolism phases: (i) 
anabolic is directed for storage; and (ii) catabolic in the storage flow to the hepatic organ for disease manifestation [8]. The evidence is inter alia fatty enlargement of the hepatic organ in diabetic children that may quickly disappear during control and treatment, even in chronic ketosis condition. As detected in high-income societies, an increase of obesity and metabolic syndrome prevalence predisposes to pathophysiological alterations which result in nonalcoholic fatty liver disorder that exacerbates acute hepatic organ derangement to concomitant cirrhosis and oncological hepatocellular degeneration [9]. Variations in insulin response, beta-oxidation, lipid transport and storage disruption, autophagy as well as established disequilibrium in chemokines and nuclear receptor signaling are implicated in these variations or alterations. Insulin, adipokines, epinephrine and other agonists stimulate pathways which regulate the activities of key enzymes of metabolism control in the integration of organismal carbohydrate and lipid metabolism. Defined overnutrition dysregulates these extant pathways with perturbative sequelae leading to insulin resistance and type 2 diabetes [9]. The pathogenesis of obesity, type 2 diabetes and insulin resistance is associated with perturbed lipid and carbohydrate metabolism. Obesity development is a complex process connected with genetic susceptibility and environmental factors that is not clearly elucidated [10]. Lipid aggregation in inflammation and ER stress is clinically evident in liver regeneration and hepatic oncological derangement [11]. The subclass of diabetes characterized as not insulin-responsive or dependent, NIDDM depicts insulin resistance and hyper-insulinaemia, with resultant glucose intolerance, hyperglycaemia and fulminant diabetes and co-morbidityn of ketosis and obesity [5].

\section{Genetic Considerations}

Obesity constitutes a significant aspect of metabolic syndrome, predisposing to type 2 diabetes development. There is exacerbation in the incidence of diabetes and metabolic syndrome with undefined aetiology for the increase in incidence. This may be influenced genetically in the prevalence of these disorders. It has not been clearly determined how genetic variables have interactions with environmental and dietary aspects to accelerate their incidence. It has been revealed that genes associated with pathways of carbohydrate, lipid and amino acid metabolism, as well as glycan biosynthesis, pathways in the metabolism of cofactors and vitamins, ubiquitin mediated proteolysis, pathways of signal transduction, interactions of neuroactive ligand-receptor, pathways of nervous system, and neurodegenerative perturbation pathways are upregulated in obesity in comparison to normal persons [6]. Obversely, genes associated with molecules of cell adhesion, cytokine-cytokine receptor interaction, insulin signaling and immune system pathways undergo downregulation in obesity. Genes implicated in signal transduction, actin cytoskeleton regulation, processing and presentation of antigen, complement and coagulation cascades, axon guidance and pathways of neurodegenerative disorders are upregulated in type 2 diabetic subjects with family history of diabetes in contradistinction to diabetic subjects devoid of any family history. Genes connected with pathways of oxidative phosphorylation, immune, nervous system and metabolic diseases are upregulated in diabetic subjects with diabetes family history, but not in diabetes devoid of any family history [6]. On the contrary, genes related in lipid and amino acid pathways, ubiquitin mediated proteolysis, signal transduction, insulin and PPAR signaling pathways are downregulated in individuals with diabetes and concomitant family history. It is evident that genes connected in inflammatory pathways are disparately expressed in both obesity and type 2 diabetes. These findings are indicative that genes associated with carbohydrate, lipid, and amino acid metabolic pathways, neuronal function and inflammation are significant in obesity and type 2 diabetes pathology. Genetic predisposition contributes immensely to obesity as determined by familial aggregation, twin and adoption studies [12, 13]. Obesity results from energy intake, mainly accumulated as triglycerides surpassing energy expenditure [14]; and influenced by age, diet, developmental stage, genes and physical activity [15]. The prevalence increase of obesity correlates with type 2 diabetes prevalence, impaired glucose tolerance [16] and numerous complications of both disorders, such as arthritis, hypertension, sleep apnoea, cardiovascular disorders and organ perturbations [17]. As a result of the increase in obesity and diabetes prevalence, it is perspicuous that future life expectancy of affected subjects is likely to decline [18].

\section{Oxidative/Respiratory Presentations}

Competition for respiration among substrates in animal tissues is depicted in the quantitative interaction between glucose and fatty acids that is evident in the Glucose Fatty Acid Cycle of the reciprocal metabolic linkage between glucose and fatty acids. The production of glucose predisposes to glucose oxidation and glucose including lipid storage, with the inhibition of fatty acid oxidation. The availability of free fatty acids predisposes to fatty acid oxidation and oxidation, with inhibition of glucose oxidation and augmentation of glucose storage during depletion of glycogen reserves [19]. Evidence exists that fatty acid oxidation inhibits glucose production in liver with defined enzyme mechanisms. A permissive latitude for fatty acids during insulin secretory response of islet beta-cells is notable as a protective mechanism in continuous availability of respiratory substrate. Extended duration in islet betacell exposure to fatty acids debilitates the insulin secretory response to glucose and presenting mechanisms. Fatty acid oxidation is associated with impairment of glucose oxidation in uncontrolled type 1 and type 2 diabetes; with paucity of information in the role of fatty acids in the depletion of glucose storage or glycogen deposition. Type 2 diabetes depicts glucose storage inhibition resulting in protracted augmentation of plasma FFA I humans and experimental animals is connected with glycogen depletion; in contrast, glucose storage inhibition in type 2 diabetes is related to glycogen reduction [19]. The defined functionality of fatty acids in perturbed carbohydrate metabolism in type 2 diabetes paves way for future research. 
Combustion pervades life, and ardent research directs itself on the prominent fuel substrates which provide sustenance, are in competition and interaction among themselves for respiratory combustion. Numerous features of imminent mutual interaction that is exhibited in both reciprocal and dependent characteristics between glucose and lipid metabolism have been elucidated [20]. These include (i) the inhibitory impacts of increased levels of fatty acids on glucose oxidation through inactivation of mitochondrial pyruvate dehydrogenase or by means of desensitization of insulin-mediated glucose transport; (ii) the inhibitory effects of augmented glucose content on fatty acid oxidation via malonyl-CoA regulation of fatty acid ingress into the mitochondria; and (iii) the stimulatory impacts of exacerbated glucose abundance on de novo lipogenesis, i.e. lipid synthesis from glucose by means of SREBP1c glycolytic and lipogenic enzyme regulation. It is posited that energy-dissipating substrate cycling linking glucose and lipid metabolism to thermogenesis functions as a modulating mechanism that controls intramyocellular lipid homeostasis culminating in contributing to skeletal muscle protection against lipotoxicity [19-23].

Lipid oxidation rate may correlate with plasma free fatty acid contents. Lipid contribution to energy expenditure is greater in obese as compared to control subjects. This is usurped by lipids in the energy metabolism of both diabetic and non-diabetic obese individuals, and it is the resultant impact of their elevated fat contents. The concomitant decline in carbohydrate metabolism ostensibly results as sequelae to variations in glucose tolerance, with resultant retarded glucose storage and oxidation in the obese subject [24]. In normal pregnancy, it has been realized that plasma triglycerides are elevated 2-3 fold. Deficiency of a significant impact of diabetes, body weight, and augmented carbohydrate intake on blood lipids during gestation differentiates pregnancy from atherosclerosisassociated hypertriglyceridaemias and relates to a distinct physiologic regulatory mechanism [25].

Carbohydrate and lipid metabolism is markedly dependent on mitochondria regulated by NRF-1 [26]. The metabolic pathways are controlled via metabolic variations at levels of high energy substances, such as AMP/ATP and the NAD+/NADH ratios. Reduced levels of ATP activate AMPK that elicits the activation of eclectic transcription factors, NRF-1, that regulates both carbohydrate and lipid metabolism. Conversely, alterations in the $\mathrm{NAD+/NADH}$ ratios influence the activity of other metabolic regulators like the sirtuins which are protein deacetylases [26]. Sirtuins augment the activity of transcriptional co-activator, PGC-1 via deacetylation, the major co-activator of NRF-1. Further research is required to determine if sirtuins directly regulate NRF-1 activity in relation to carbohydrate and lipid metabolism.

\section{Management and Control}

Dietary approaches and strategies are pertinent in diabetes and obesity management. Stringent dietary regulation with minimal carbohydrate diet has been extensively applied, that later subsided with novel applications of medications. The major objective has been to ameliorate dietary fat intake in order to obviate the atheriosclerotic disease risk, with less attention on carbohydrate quality and abundance. With exacerbating obesity and diabetes globally, the trend and target are on the diet micronutrient composition. Low carbohydrate diets have been effective in initial weight mitigation and glycaemic control improvement, with long-run acceptance and deteriorating lipid profile [27]. Modulations of extremely reduced carbohydrate contents involve restricting saturated fat and augmenting both carbohydrate and lipid. Decreasing saturated fat is ostensibly the key to reduce low-density lipoprotein, LD2 cholesterol with retardation of adverse impacts of conventional very low carbohydrate diets. Augmented dietary protein predisposes satiety, decreased energy intake and concomitant improved glycaemic homeostasis, but devoid of substandard improvement in glycaemic regulation or cardiovascular sequelae regarding the impact of weight loss. Type 1diabetes has responded to regulatory effects with low carbohydrate diets, without ketosis and hypoglycaemic complications. Carbohydrate-restricted diets exhibit effective weight reduction as low fat diets; and fat substitution for carbohydrate is invariably advantageous concerning cardiovascular disease risk without need for weight loss; and improves metabolic syndrome characteristics [28]. Low carbohydrate diets are dependent upon mechanisms of glucose directly or indirectly via insulin that is a major regulatory component in gluconeogenesis, glycogen metabolism, lipogenesis and lipolysis.

Studies have assessed the relative impact of fat compared to carbohydrate and the distinctiveness among fatty acids and variants of carbohydrates on insulin resistance and related risk factors for diabetes, cardiovascular disease and obesity. The fibre content of the carbohydrate diet ostensibly provides advantages regarding diabetic control, while lower cholesterol and postprandial blood glucose are connected with viscous fibres [29]. Resting energy expenditure and measurement of both carbohydrate and lipid oxidation rates as well as measurements observed in both fasting and parenterally fed states were determined [7]. Although, there were no variations in resting energy expenditure, a significant decrease $\mathrm{p}<0.05$ in RQ $5 \%$ and carbohydrate oxidation rate, $24 \%$ accompanied by a concomitant exacerbation of lipid oxidation rate, $42 \%$ was detected in parenteral nutrition in fusion. There were no disparities in plasma insulin, glucose, and insulin resistance observed in pre- and post-infliximab (9). Improvements in insulin resistance following anti-TNF-alpha therapy have been observed in inflammatory states in children, but no alterations were detected in adult patients presenting with Chron's disease [30].

A study of the interactions between fasting plasma glucose and fasting insulin levels and diets with disparate carbohydrate concentrations indicated that fasting plasma glucose and insulin are strong predictors of weight dissipation response to diets with disparate micronutrient contents and important for customized weight management [31]. It has also been shown that any diet type manifesting decreased energy intake culminates in weight dissipation and associated commendable metabolic and functionality changes with long-run prevention of type 2 diabetes progression [32].

There is growing evidence that dietary plant polyphenols are potential nutraceuticals and supplementary treatments for type 2 diabetes due to their biological attributes, such as high concentration of phytochemicals and 
antioxidant capacity [33] Findings from in vitro, animal models and human experiments indicated that plant-food polyphenols, and polyphenol-rich products (i) attenuate dyslipidaemia, hyperglycaemia and insulin resistance, (ii) potentiate adipose tissue metabolism, (iii) mitigate oxidative stress and stress-sensitive signaling pathways and inflammatory responses [34], as well as (iv) modulate carbohydrate and lipid metabolism. Polyphenols can contribute to the prevention and abatement of long-term diabetic sequelae, such as cardiovascular disorder, nephropathy, retinopathy and other predisposing factors $[35,36]$.

\section{Discussion}

Diabetes constitute a metabolic disorder characterized by chronic hyperglycaemia and immune-mediated type 1 diabetes, insulin-resistant type 2 diabetes, gestational, genetic, environmental, infectious, behavioural or druginduced impairments [4] It is generally postulated that obesity and type 2 diabetes have escalated in prevalence globally with concomitant sequelae or complications in morbidity and mortality. The pathophysiology or pathobiology of these conditions have been difficult to unravel or elucidate. Several hormone-like signaling agents which influence energy metabolism are emitted from adipocytes, such as leptin, as well as immature cells, such as tumour necrosis factor-alpha. Obesity and type 2 diabetes present marked gene-environment interaction [37]. Paucity of data is extant on the single-gene defects which result in these states, but in several instances, polygenic contributions have been detected [2, 38, 39].

\section{Conclusion}

Numerous environmental states including foetal ambience, lifestyle and social diversions have been implicated in the predisposition of these ailments or disorders, namely diabetes and obesity. Fundamentally, an imbalance between caloric ingress and energy dissipation elicits endocrine and/or metabolic responses exacerbating the agedependent dysregulation in lipid and carbohydrate metabolism.

\section{References}

[1] Uranga, R. M. and Keller, J. L., 2019. "The complex interactions between obesity, metabolism and the brain. ." Front Neurosci, Available: https://doi.org/10.3389/fnins.2019.00513

[2] Chukwuma, S. C. and Tuomilehto, J., 1998. "The "thrifty" hypotheses: clinical and epidemiological significance for non-insulin-dependent diabetes mellitus and cardiovascular disease risk factors." $J$. Cardiovasc Risk., vol. 5, pp. 11-23.

[3] Chukwuma Sr, C., 2018. "Type 1 diabetes mellitus: issues, challenges and opportunities." Edelweiss Publications Applied Science, vol. 2, pp. 290-297.

[4] Baynes, H. W., 2015. "Classification, pathophysiology, diagnosis and management of diabetes mellitus." $J$. Diabetes Metab, vol. 6, p. 541.

[5] Candib, L. M., 2007. "Obesity and diabetes in vulnerable populations: reflection and proximal causes." Ann. Fam. Med., vol. 5, pp. 547-556.

[6] Das, U. N. and Rao, A. A., 2007. "Gene expression profile in obesity and type 2 diabetes mellitus." Lipids in Health and Disease, vol. 6, p. 35

[7] Kim, J. B., 2016. "Dynamic cross talk, between metabolic organs in obesity and metabolic diseases." Exp. Mol. Med., vol. 48, p. e214.

[8] Lawrence, R., D., 1941. "Interactions of fat and carbohydrate metabolism - Aspects and therapies." Proc. R. Soc. Med., vol. 35, pp. 1-10.

[9] Hardie, D., G., 2012. Organismal carbohydrate and lipid homeostasis. Cold Spring Harbor Perspectives in Biology.

[10] Musso, G., Gambino, R., and Cassader, M., 2010. "Obesity, diabetes and gut microbiota. The hygiene hypothesis expanded." Diabetes Care, vol. 33, pp. 2277-2284.

[11] Bechmann, L. P., Hannivoort, R. A., and Gerken, G., 2012. "The interaction of hepatic lipid and glucose metabolism in liver diseases." J. Hepatology., vol. 56, pp. 952-964.

[12] Bouchard, C. and Tremblay, A., 1997. "Genetic influences on the response of body fat distribution to positive and negative energy balances in human identical twins." Nutr., vol. 127, pp. 943S-947S.

[13] Stunkard, A. J., Harris, J. R., Pedersen, N. L., and McLearn, G. E., 1990. "The body mass index of twins who have been reared apart." N. Engl. J. Med., vol. 332, pp. 1483-1487.

[14] Bray, G. A., 2004. "Medical consequences of obesity." J. Clin. Endocrinol. Metab., vol. 89, pp. $2583-2589$.

[15] Kopelman, P. G., 2000. "Obesity as a medical problem." Nature, vol. 404, pp. 635-643.

[16] Harris, M. I., 1998. "Diabetes in America: epidemiology and scope of the problem." Diabetes Care, vol. 21, pp. C11-C14.

[17] Singla, P., Bardoloi, A., and Parkash, A. A., 2010. "Metabolic effects of obesity: A review." World J. Diabetes, vol. 1, pp. 76-88.

[18] Olshansky, S. J., Passaro, D. J., C., H. P., Layden, J., Carnes, B., and Brody, J. A., 2005. "A potential decline in life expectancy in the United States in the 21st century." N. Engl. J. Med., vol. 352, pp. 11381145 .

[19] Randle, P. J., 1998. "Regulatory interactions between lipids and carbohydrates: the glucose fatty acid cycle after 35 years." Diab. Metab. Res. Rev., vol. 14, pp. 263-283. 
[20] Dulloo, A. G., Gubler, M., and Montani, J. P., 2004. "Substrate cycling between de novo lipogenesis, and lipid oxidation: A thermogenic mechanism against skeletal muscle lipotoxicity and glucolipotoxicity." In Proceedings of the Second Fribourg Obesity Research Conference (FORC-2003). Int J Obesity. pp. S29S37.

[21] Ferrannini, E., Barrett, E. J., Bevilacqua, S., and DeFronzo, R. A., 1983. "Effects of fatty acids on glucose production and utilization in man." J. Clin. Invest., vol. 72, pp. 1737-1747.

[22] Frayn, K. N., 2003. "The glucose-fatty acid cycle: a physiologic perspective." Biochem Soc. Trans., vol. 31, pp. 1115-1119.

[23] Kelley, D. E., Mokan, M., Simoneau, J. A., and Mandarino, L. J., 1993. "Interaction between glucose and free fatty acid metabolism in human skeletal muscle." J. Clin. Invest., vol. 92, pp. 91-98.

[24] Golay, A., Felber, J. P., and Meyer, H. U., 1984. "Study on lipid metabolism in obesity diabetes." Metabolism, vol. 33, pp. 111-116.

[25] Warth, M. R. and Knopp, R. H., 1977. "Metabolism in pregnancy: V. Interactions of diabetes, body weight, age, and high carbohydrate diet." Diabetes, vol. 26, pp. 1056-1062.

[26] Ferreira, Z., Ayeleso, A., and Mukwevh, O. E., 2014. "Control of carbohydrate and lipid metabolism by NRF-1 and sirtuins: Implications on type 2 diabetes and obesity." Chem. Biol. Lett., vol. 1, Available: https://www.researchgate.net/publication/325367790_Control_of_carbohydrate_and_lipid_metabolism_by NRF-1_and sirtuins_Implications on type_2_diabetes and obesity

[27] Hall, R. M., Strong, A. P., and Krebs, J. D., 2016. "Importance of low carbohydrate diets in diabetes management." Nutr. Diet. Suppl., vol. 8, pp. 9-19.

[28] Beinstein, D. R., 2008. "Dietary carbohydrate restriction in type 2 diabetes and metabolic syndrome: time for a critical appraisal." Nutrition and Metabolism, Available: http://www.diabetes.book.com/dietarycarbohydrate-restriction/

[29] Hung, T., Sievenpiper, J. L., and Marchie, A., 2003. "Fat versus carbohydrate in insulin resistance, obesity, diabetes and cardiovascular disease." Curr. Opin. Clin. Nutr. Metab. Care., vol. 6, pp. 165-176.

[30] Steiner, S. J., Pfefferkorn, M. D., Fitzgerald, J. F., and Denne, S. C., 2008. "Carbohydrate and lipid metabolism following infliximab therapy in pediatric Chron's Disease." Pediatric Research, vol. 64, pp. 673-676.

[31] Hjorth, M. F., Astrup, A., Zohar, Y., Urban, L. E., Sayer, R. D., and Patterson, B. W., 2019. "Personalized nutrition: pretreatment glucose metabolism determines individual long-term weight loss responsiveness in individuals with obesity on low-carbohydrate versus low-fat diet." Int. J. Obes., vol. 43, pp. 237-44.

[32] Browns, F., 2018. "Overweight and diabetes prevention: is a low-carbohydrate-high-fat diet recommendable?" European. J. Nutr., vol. 57, pp. 1301-12.

[33] Bahadoran, Z., Mirmiran, P., and Azizi, F., 2013. "Dietary polyphenols as potential nutraceuticals in management of diabetes: A review." J. Diab. Metab. Disorders, vol. 12, p. 43.

[34] Evans, J. L., Goldfine, I. D., Maddux, B. A., and Grodsky, G. M., 2002. "Oxidative stress and stressactivated signaling pathways: a unifying hypothesis of type 2 diabetes." Endocr Rev., vol. 23, pp. 599-622.

[35] Bahadoran, Z., Golzarand, M., Mirmiran, P., Saadati, N., and Azizi, F., 2012. "The association of dietary phytochemical index and cardiometabolic risk factors in adults: Tehran lipid and glucose study." Nutri. Metab. (Lond), vol. 9, p. 70.

[36] Pandey, K. B. and Rizvi, S. I., 2009. "Plant polyphenols as dietary antioxidants in human health and disease." Oxid Med Cell Longev, vol. 2, p. 7.

[37] Chukwuma Sr, C., 2014. "Is diabetes a model for gene-environment interaction in premature senescence? ." J. A. B. H., Available: www.iiste.org/Journals/index.php/JBAH/article/view/17380

[38] Brown, M. A., Storlein, L. H., and Huang, X.-F., 2010. Dietary fat and carbohydrate composition metabolic diseases. Chapter 21. In: Montmayeur, $j, p$. And le coutre, j. (eds). Fat detection, task, texture and post-ingestive effects. Boca Raton (FC): CRC Press Taylor and Francis.

[39] Romao, I. and Roth, J., 2008. "Genetic and environmental interactions in obesity and type 2 diabetes." $J$. Am. Dietetic Assoc., vol. 108, pp. S24-S28. 